\title{
Associations among bronchioloalveolar carcinoma components, positron emission tomographic and computed tomographic findings, and malignant behavior in small lung adenocarcinomas
}

Morihito Okada, MD, PhD, ${ }^{a}$ Shunsuke Tauchi, MD, ${ }^{a}$ Koichiro Iwanaga, MD, ${ }^{a}$ Takeshi Mimura, MD, ${ }^{a}$ Yoshitaka Kitamura, MD, ${ }^{a}$ Hirokazu Watanabe, MD, PhD, ${ }^{\text {b }}$ Shuji Adachi, MD, PhD, ${ }^{\text {b }}$ Toshiko Sakuma, MD, PhD, ${ }^{c}$ and Chiho Ohbayashi, $\mathrm{MD}, \mathrm{PhD}^{\mathrm{c}}$

From the Departments of Thoracic Sur gery, ${ }^{\mathrm{a}}$ Radiology, ${ }^{\mathrm{b}}$ and Pathology, ${ }^{\mathrm{c}}$ Hyogo Medical Center for Adults, Akashi City, Hyogo, Japan.

Received for publication Dec 12, 2006; revisions received Feb 2, 2007; accepted for publication Feb 19, 2007.

Address for reprints: Morihito Okada, MD, $\mathrm{PhD}$, Department of Surgical Oncology, Hiroshima University, 1-2-3 Kasumi Minami-Ku, Hiroshima City 734-8551, Japan (E-mail: morihito1217jp@aol.com).

J Thorac Cardiovasc Surg 2007;133:1448-54 $0022-5223 / \$ 32.00$

Copyright $\odot 2007$ by The American Association for Thoracic Surgery

doi:10.1016/j.jtcvs.2007.02.023
Objective: The aggressiveness of small adenocarcinomas has not been fully evaluated using integrated positron emission tomography/computed tomography. We investigated malignant aggressiveness according to positron emission tomography/computed tomography, high-resolution computed tomographic findings, and the proportions of pathologically defined bronchioloalveolar carcinomas in cT1NOM0 lung adenocarcinoma.

Methods: Sixty consecutive patients with cT1N0M0 lung adenocarcinomas of $3 \mathrm{~cm}$ or less in diameter underwent fluorodeoxyglucose-positron emission tomograph/computed tomography, and high-resolution computed tomography, followed by complete tumor resection. Correlations between the proportion of bronchioloalveolar carcinoma and maximum standardized uptake value on positron emission tomographic scan/computed tomographic scan, ground-glass opacity, and tumor shadow disappearance rate were investigated and the findings were compared with clinicopathologic features.

Results: Lymphatic and vascular invasion occurred in 18 (30\%) and 13 (22\%) patients, respectively, whereas hilar or mediastinal lymph nodes occurred in 8 patients $(13 \%)$. Maximum standardized uptake value generally seemed the most valuable predictor of lymphatic invasion, vascular invasion, and nodal metastasis compared with ground-glass opacity, tumor shadow disappearance rate, and bronchioloalveolar carcinoma ratios. Although the association was significant between the bronchioloalveolar carcinoma ratio versus maximum standardized uptake value, ground-glass opacity ratio, and tumor shadow disappearance rate (all $P<.0001$ ), maximum standardized uptake value $\left(R^{2}=0.245\right)$ was less correlated with the bronchioloalveolar carcinoma ratio than was the ground-glass opacity ratio $\left(R^{2}=\right.$ $0.554)$ and tumor shadow disappearance rate $\left(R^{2}=0.671\right)$.

Conclusions: The malignant behavior of small adenocarcinomas with a lower maximum standardized uptake value and a greater proportion of ground-glass opacity, tumor shadow disappearance rate, and bronchioloalveolar carcinoma was less aggressive. Maximum standardized uptake value was a more powerful clinical predictor of biologic tumor performance, independent of pathologic bronchioloalveolar carcinoma proportion. Preoperative assessment of maximum standardized uptake value on positron emission tomographic/computed tomographic findings, in addition to the ground-glass opacity ratio and tumor shadow disappearance rate on high-resolution computed tomographic scans, might be useful to guide treatment strategies for small adenocarcinomas.

$\mathrm{R}$ ecent improvements in imaging technology and the widespread use of computed tomographic (CT) scans for screening have increased the probability of detecting small-sized lung cancers, especially adenocarcinomas, and this has led to concerns over whether some of these cancers can be sufficiently 


$$
\begin{aligned}
& \text { Abbreviations and Acronyms } \\
& \begin{aligned}
\text { BAC } & =\text { bronchioloalveolar carcinoma } \\
\text { CEA } & =\text { serum carcinoembryonic antigen } \\
\text { GGO } & \text { ground-glass opacity } \\
\text { HR-CT } & =\text { high-resolution computed tomography } \\
\text { HU } & =\text { Hounsfield units } \\
\operatorname{maxD} & =\text { maximum dimensions of the tumors } \\
\text { perD } & =\text { largest dimension perpendicular to the } \\
\text { PET } & \text { maximum axis } \\
\text { SUV } & =\text { standardized uptake value } \\
\text { TDR } & =\text { tumor shadow disappearance rate }
\end{aligned}
\end{aligned}
$$

treated by sublobar resection as an alternative to lobectomy. ${ }^{1,2}$ In fact, about $20 \%$ of clinical $\mathrm{T} 1$ cancers have nodal involvement and the remainder follows a more indolent biologic course. Because the risk of nodal involvement and subsequent systemic metastasis is not absolutely linked to tumor size, ${ }^{3}$ the preoperative ability to distinguish biologically aggressive from indolent tumors is extremely important for an indication of sublobar resection.

On high-resolution computed tomographic (HR-CT) scanning, ground-glass opacity (GGO), defined as a misty component in lung attenuation, and tumor shadow disappearance rate (TDR), defined as the ratio of tumor area of the mediastinal window to that of the lung window, seem linked to adenocarcinoma aggressiveness and thus to the risk of nodal involvement. ${ }^{4,5}$ In addition, bronchioloalveolar carcinomas (BACs) typified by lepidic growth along alveoli without invasive areas are indolent and basically expressed as GGO or areas of tumor shadow disappearance on HR-CT.

On the other hand, integrated positron emission tomography (PET)/CT with F-18 fluorodeoxyglucose (FDG) is a refined type of dedicated FDG-PET that measures a standardized uptake value (SUV). This is an increasingly useful noninvasive imaging modality with which to evaluate indeterminate lung nodules, regional lymph nodes, distant metastases, tumor invasiveness, and responses to chemoradiotherapy. ${ }^{6-14}$ Furthermore, FDG-PET/CT might be able to quantify the aggressiveness of small adenocarcinomas.

Notably, preoperative imaging findings with PET/CT and HR-CT must be defined and correlated with pathologic findings. Understanding the biologic features of small adenocarcinomas preoperatively could provide clues to select patients for radical sublobar resection. We therefore examined correlations between SUV, GGO proportion, and TDR with the BAC proportion defined on pathologic sections of surgical specimens. The results of this study could improve approaches to diagnosing and treating small lung adenocarcinomas that might become a more vital part of thoracic surgical tradition.

\section{Materials and Methods}

Between October 2005 and September 2006, the same surgical team completely resected tumors and assessed ipsilateral hilar and mediastinal lymph nodes for primary cT1N0M0 lung adenocarcinomas ( $3 \mathrm{~cm}$ or less in diameter) in 60 consecutive patients. Our institutional review board approved the prospective database used in this retrospective analysis. Surgical-pathologic staging was performed according to the New International Staging System for Lung Cancer. ${ }^{15}$ All patients underwent integrated FDG-PET/CT and HR-CT within 1 month before resection. Patients with diabetes were excluded from this study.

FDG-PET/CT scans were carried out on an integrated PET/CT scanner (Discovery ST8 PET/CT Scanner; General Electric, Milwaukee, Wis). Patients were requested to fast for 4 hours and then intravenously received $185 \mathrm{MBq}(5 \mathrm{mCi})$ of $\mathrm{FDG}$, followed by PET scanning after 40 minutes. Iterative reconstruction with CT attenuation correction was performed. Also, chest CT scan was available for visual correlation. Maximum SUV was established by drawing regions of interest on attenuation-corrected FDG-PET images around the primary tumor and calculated by the software within the PET/CT scanner using the following formula: Maximum SUV $=[C(\mu \mathrm{Ci} / \mathrm{mL}) / I D(\mu \mathrm{Ci})] / w$, where $C$ is defined as activity at a pixel within the tissue identified by an regions of interest and $I D$ is defined as the injected dose per kilogram of the patient's body weight $(w)$. We adopted maximum SUV in the present analysis because it is less variable than mean SUV in measuring ${ }^{16}$ and established a value of $2.5 \mathrm{~g} / \mathrm{mL}$ or higher as positive.

Contrast-enhanced HR-CT was performed on a multidetector CT capable of generating $16 \times 0.5-\mathrm{mm}$ slices (Aquilion $16 \mathrm{SH}$; Toshiba Medical Systems, Tokyo, Japan). Slices with 1-mm spaces were taken through the tumor in addition to all slices with 6-mm spacing obtained from the apex of the lung to the base. The images were photographed using a window level of -600 Hounsfield units (HU) with a window width of $1700 \mathrm{HU}$ (lung windows) and a level of $25 \mathrm{HU}$ with a width of $350 \mathrm{HU}$ (mediastinal windows). TDR and GGO were assessed by independent observers, and discrepancies in evaluation among them were resolved by averaging their determined values. The observers measured the maximum dimensions of the tumors (maxD) and the largest dimension perpendicular to the maximum axis (perD) on both the lung and mediastinal windows. As reported elsewhere, ${ }^{4,5,17}$ TDR was defined as follows: $\operatorname{TDR}(\%)=[1-(\operatorname{maxD} \times$ perD on mediastinal windows $/$ maxD $\times$ perD on lung windows $)] \times$ 100.

The tumors resected surgically were fixed in $10 \%$ formalin and embedded in paraffin. The sections including the largest cut were stained with hematoxylin-eosin and elastica van Gieson for histopathologic examination. The estimation regarding the proportion of BAC areas relative to the whole tumors was performed by independent pathologists in the same manner as that for PET/CT and HR-CT reviews. The clinical records of all patients were also reviewed to determine age, gender, smoking status, serum carcinoembryonic antigen (CEA) level, tumor size, operative procedure, lymph node status, lymphatic invasion, and vascular invasion. When lymphatic and blood vessels had been invaded, adenocarcinoma cells were histopathologically identifiable in the lymphatic and blood vessel lumen, respectively. Lymphatic and 
TABLE 1. Clinical characteristics of patients with cT1NOMO adenocarcinoma relative to the level of maximum SUV on FDG-PET/CT images

\begin{tabular}{lccc}
\hline & $\begin{array}{c}\text { Maximum SUV } \\
\mathbf{5} \mathbf{2 . 5}(\mathbf{n}=\mathbf{3 9})\end{array}$ & $\begin{array}{c}\text { Maximum SUV } \\
\mathbf{>} \mathbf{2 . 5}(\mathbf{n}=\mathbf{2 1})\end{array}$ & $\boldsymbol{P}$ value \\
\hline Age (y) (means \pm SD) & $64.3 \pm 9.8$ & $66.1 \pm 9.2$ & .4939 \\
Gender & & & \\
$\quad$ Male & 17 & 12 & .4184 \\
$\quad$ Female & 22 & 9 & \\
Smoking status & & & \\
$\quad$ Smoker & 18 & 13 & .2874 \\
$\quad$ Nonsmoker & 21 & 8 & \\
CEA (ng/mL) & & & \\
$\quad \leq 5$ & 35 & 13 & .0169 \\
$\quad>5$ & 4 & 8 & \\
Tumor size (mm) & & & \\
$\quad$ T $\leq 20$ & 25 & 5 & .0061 \\
$\quad 20<$ T $\leq 30$ & 14 & 16 & \\
Procedure & & & \\
$\quad$ Lobectomy & 27 & 17 & .3767 \\
$\quad$ Segmentectomy & 12 & 4 & \\
\hline
\end{tabular}

SUV, Standardized uptake value; FDG-PET/CT, positron emission tomography-computed tomography with F-18 fluorodeoxyglucose; $S D$, standard deviation; CEA, carcinoembryonic antigen.

blood vessels were identified based on morphology using light microscopy and elastica stain. Peritumoral invasion (observed outside the margin of the invasive carcinoma) and intratumoral types of invasion were assessed.

The associations between clinical factors and maximum SUV, GGO ratio, TDR, and BAC ratio were evaluated using the $\chi^{2}$ test or the Fisher exact tests. To elucidate variables for the prediction of nodal, lymphatic, and vessel involvement, we performed logistic regression analyses. To further clarify independent variables in relation to the prediction of nodal involvement, we performed multiple logistic regression analyses using gender for category and age, CEA, maximum SUV, GGO ratio, TDR, and BAC ratio as continuous variables. We also examined which of maximum SUV, GGO ratio, or TDR correlated more closely with the BAC ratio using a correlation coefficient.

\section{Results}

Of 60 patients examined, 31 (52\%) were women and 29 were men. The mean age was 65 years (range $34-82$ years). Thirty-one patients (52\%) were smokers, of whom 17 currently smoked, and 29 had never smoked. Forty-eight patients $(80 \%)$ had a normal preoperative CEA level, the upper limit of which was $5.0 \mathrm{ng} / \mathrm{mL}$. Half of the patients had a primary tumor with a diameter of $20 \mathrm{~mm}$ or less, and the other half had a tumor of 21 to $30 \mathrm{~mm}$ in diameter. Lobectomy was the most frequently applied type of resection $(\mathrm{n}=$ $44,73 \%$ ), and 16 patients underwent segmental resections. The reasons for sublobar resection included radical segmentectomy for tumors $2 \mathrm{~cm}$ or smaller in 11 patients and compromised reserve in 5. Pneumonectomy was never per-
TABLE 2. Clinical characteristics of patients with cT1NOMO adenocarcinoma relative to the proportion of GGO on HR-CT images

\begin{tabular}{lccr}
\hline & $\begin{array}{c}\text { GG0 } \geq \mathbf{5 0} \% \\
(\mathbf{n}=\mathbf{2 0})\end{array}$ & $\begin{array}{c}\text { GG0 }<\mathbf{5 0} \% \\
(\mathbf{n}=\mathbf{4 0})\end{array}$ & $\boldsymbol{P}$ value \\
\hline Age (y) (means $\pm \mathrm{SD})$ & $65.4 \pm 8.9$ & $64.1 \pm 10.9$ & .6367 \\
Gender & & & \\
$\quad$ Male & 7 & 22 & .1772 \\
$\quad$ Female & 13 & 18 & \\
Smoking status & & & \\
$\quad$ Smoker & 8 & 23 & .2749 \\
$\quad$ Nonsmoker & 12 & 17 & \\
CEA (ng/mL) & & & \\
$\quad \leq 5$ & 19 & 29 & .0466 \\
$\quad>5$ & 1 & 11 & \\
Tumor size (mm) & & & \\
$\quad$ T $\leq 20$ & 13 & 17 & .1702 \\
$20<$ T $\leq 30$ & 7 & 23 & \\
Procedure & & & \\
$\quad$ Lobectomy & 14 & 30 & .7604 \\
$\quad$ Segmentectomy & 6 & 10 & \\
\hline
\end{tabular}

GGO, Ground-glass opacity; $H R-C T$, high-resolution computed tomography; $S D$, standard deviation; CEA, carcinoembryonic antigen.

formed, and wedge resection was excluded because nodal status could not be histopathologically confirmed. All patients had curative R0 resections. Lymphatic and vascular invasion occurred in $18(30 \%)$ and $13(22 \%)$ patients, respectively. Fifty-two patients $(87 \%)$ had no nodal involvement, whereas hilar or mediastinal lymph node involvement was found in 8 patients (13\%).

The clinical features of the patients in relationship to maximum SUV on PET/CT are summarized in Table 1. Larger tumors $(P=.0061)$ and a high preoperative CEA value $(P=.0169)$ were more frequent in patients with a positive maximum SUV. Maximum SUV did not differ with age, gender, smoking status, and operative procedure. The CEA value was frequently high in patients whose proportions of GGO and TDR on HR-CT and pathologic BAC on surgical specimen (Tables 2, 3, and 4) were below 50\% proportion $(P=.0446, P=.0498$, and $P=.0253$, respectively).

We then analyzed the predictors for lymphatic invasion, vascular invasion, and nodal metastasis (Table 5). Maximum SUV, GGO proportion, TDR, and BAC proportion were all found to be significant predictors of lymphatic $(P<$ $.0001, P=.0189, P=.0020$, and $P=.0005$, respectively) and vascular $(P<.0001, P=.0433, P=.0256$, and $P<$ .0001 , respectively) invasion. Whereas maximum SUV, TDR, and BAC proportion were significant predictors of nodal metastasis $(P=.0019, P=.0201$, and $P=.0053$, respectively), the GGO proportion was not significantly useful for predicting nodal status $(P=.2487)$. Overall, 
TABLE 3. Clinical characteristics of patients with cT1NOMO adenocarcinoma relative to TDR on HR-CT images

\begin{tabular}{lccc}
\hline & $\begin{array}{c}\text { TDR } \geq \mathbf{5 0 \%} \\
(\mathbf{n}=\mathbf{3 2})\end{array}$ & $\begin{array}{c}\text { TDR }<\mathbf{5 0 \%} \\
(\mathbf{n}=\mathbf{2 8})\end{array}$ & $\boldsymbol{P}$ value \\
\hline Age (y) (means $\pm \mathrm{SD})$ & $66.1 \pm 9.5$ & $63.9 \pm 9.6$ & .3632 \\
Gender & & & \\
$\quad$ Male & 13 & 16 & .3005 \\
$\quad$ Female & 19 & 12 & \\
Smoking status & & & \\
$\quad$ Smoker & 16 & 15 & .8017 \\
$\quad$ Nonsmoker & 16 & 13 & \\
CEA (ng/mL) & & & \\
$\quad \leq 5$ & 29 & 19 & .0498 \\
$\quad>5$ & 3 & 9 & \\
Tumor size (mm) & & & \\
$\quad$ T $\leq 20$ & 20 & 10 & .0692 \\
$\quad 20<$ T $\leq 30$ & 12 & 18 & \\
Procedure & & & \\
$\quad$ Lobectomy & 21 & 23 & .2417 \\
$\quad$ Segmentectomy & 11 & 5 & \\
\hline
\end{tabular}

$T D R$, Tumor shadow disappearance rate; $H R-C T$, high-resolution computed tomography; $S D$, standard deviation; $C E A$, carcinoembryonic antigen.

maximum SUV, BAC ratio, TDR, and the GGO ratio in that order can likely reflect tumor aggressiveness. A multivariate analysis to determine independent predictors of pathologic nodal status (Table 6) revealed that age, gender, and CEA were not significant in any model. Maximum SUV $(P=$ $.0199)$, TDR $(P=.0136)$, and BAC ratio $(P=.0375)$ were significantly useful predictors of nodal status, although GGO ratio and nodal status correlated marginally but not significantly $(P=.0800)$.

Last, we performed logistic regression analyses to clarify which radiographic parameter of maximum SUV, GGO ratio, or TDR correlated better with a pathologic BAC ratio (Figure 1). Although a significant correlation was found between BAC ratio versus maximum SUV, GGO ratio, and TDR (all $P<.0001)$, maximum SUV $\left(R^{2}=0.245\right)$ had a poorer association with BAC ratio compared with GGO ratio $\left(R^{2}=0.554\right)$ and $\operatorname{TDR}\left(R^{2}=0.671\right)$. These data demonstrated that both TDR and GGO ratio on HR-CT were well correlated with pathologic BAC ratio and that maximum SUV on PET/CT had a much lesser impact as a preoperative indictor of pathologic BAC proportion.

\section{Discussion}

Although the proportion of the BAC component can mirror the malignant grade of small adenocarcinoma, ${ }^{4,18,19}$ it is defined on postsurgical specimens. Because the parts indicating GGO or TDR on HR-CT seemed to closely correlate with BAC area, the proportion of GGO and TDR might be linked to tumor aggressiveness and subsequently to risk for nodal metastases and survival. ${ }^{4,5,17}$ Characterizing and
TABLE 4. Clinical characteristics of patients with cT1NOMO adenocarcinoma relative to the proportion of $B A C$ on pathologic examination

\begin{tabular}{lccr}
\hline & $\begin{array}{c}\text { BAC } \geq \mathbf{5 0} \% \\
(\mathbf{n}=\mathbf{2 8})\end{array}$ & $\begin{array}{c}\text { BAC }<\mathbf{5 0 \%} \\
(\mathbf{n}=\mathbf{3 2})\end{array}$ & $\boldsymbol{P}$ value \\
\hline Age (y) (means \pm SD) & $65.4 \pm 10.9$ & $64.5 \pm 8.4$ & .7305 \\
Gender & & & \\
$\quad$ Male & 10 & 19 & .0773 \\
$\quad$ Female & 18 & 13 & \\
Smoking status & & & \\
$\quad$ Smoker & 12 & 19 & .3005 \\
$\quad$ Nonsmoker & 16 & 13 & \\
CEA (ng/mL) & & & \\
$\quad \leq 5$ & 26 & 22 & .0253 \\
$\quad>5$ & 2 & 10 & \\
Tumor size (mm) & & & \\
$\quad$ T $\leq 20$ & 18 & 12 & .0692 \\
$20<$ T $\leq 30$ & 10 & 20 & \\
Procedure & & & \\
$\quad$ Lobectomy & 20 & 24 & .7780 \\
$\quad$ Segmentectomy & 8 & 8 & \\
\hline
\end{tabular}

$B A C$, Bronchioloalveolar carcinoma; $S D$, standard deviation; $C E A$, carcinoembryonic antigen.

quantifying these findings on HR-CT, especially GGO, have been relatively subjective as interobserver discrepancies might have affected visual estimations.

Cerfolio and colleagues ${ }^{11}$ reported that maximum SUV of a non-small cell lung cancer nodule on dedicated PET is an independent predictor of tumor characteristics for stage, recurrence, and survival. However, the utility of FDG$\mathrm{PET} / \mathrm{CT}$ in predicting the biologic features of small adenocarcinomas, especially BACs, remains obscure. Although approximately $85 \%$ of non-small cell lung cancers are FDG positive, roughly $50 \%$ of adenocarcinomas with a BAC component are FDG positive, and pure BAC is more likely to escape detection with FDG-PET. ${ }^{20,21}$ Lower metabolic activity in adenocarcinomas containing a BAC component is assumed to be secondary to the slower pace of BAC proliferation compared with other adenocarcinomas. If higher metabolic activity is linked to more tumor aggressiveness, FDG avidity (maximum SUV) might identify a group of adenocarcinomas that is more likely to have highgrade malignant behavior.

In the present series, adenocarcinoma with a high maximum SUV as well as a low percentage of GGO, TDR, or BAC was more frequently associated with an increase in the serum CEA level. Presurgical serum CEA values are important in identifying patients at high risk of potential advanced disease and poor survival and whose specificity is higher for adenocarcinoma than squamous cell carcinoma. ${ }^{22,23}$ The present study also uncovered an even closer relationship between maximum SUV and lymphatic, blood 
TABLE 5. Univariate analysis of predictors for Ly factor, $\mathbf{V}$ factor, and $\mathbf{N}$ factor status

\begin{tabular}{|c|c|c|c|c|}
\hline Factors & Favorable & Unfavorable & Odds ratio $(95 \% \mathrm{CI})$ & $P$ value \\
\hline \multicolumn{5}{|l|}{ For Ly factor } \\
\hline Maximum SUV & $\leq 2.5$ & $>2.5$ & $17.50(4.420-69.283)$ & $<.0001$ \\
\hline GGO ratio & $\geq 50$ & $<50$ & $6.00(1.221-9.481)$ & .0189 \\
\hline TDR & $\geq 50$ & $<50$ & $7.00(1.940-25.255)$ & .0020 \\
\hline Pathologic BAC ratio & $\geq 50$ & $<50$ & $13.00(2.634-64.163)$ & .0005 \\
\hline \multicolumn{5}{|l|}{ For $\mathrm{V}$ factor } \\
\hline Maximum SUV & $\leq 2.5$ & $>2.5$ & - & $<.0001$ \\
\hline GG0 ratio & $\geq 50$ & $<50$ & $8.142(0.9759-67.947)$ & .0433 \\
\hline TDR & $\geq 50$ & $<50$ & $5.37(1.301-22.172)$ & .0256 \\
\hline Pathologic BAC ratio & $\geq 50$ & $<50$ & - & $<.0001$ \\
\hline \multicolumn{5}{|l|}{ For $\mathrm{N}$ factor } \\
\hline Maximum SUV & $\leq 2.5$ & $>2.5$ & $19.00(2.141-168.660)$ & .0019 \\
\hline GGO ratio & $\geq 50$ & $<50$ & $4.03(0.460-35.299)$ & .2487 \\
\hline TDR & $\geq 50$ & $<50$ & $10.33(1.183-90.260)$ & .0201 \\
\hline Pathologic BAC ratio & $\geq 50$ & $<50$ & - & .0053 \\
\hline
\end{tabular}

LY factor, Lymphatic invasion status; $N$ factor, lymph node status; $V$ factor, vascular invasion status; $C l$, confidence interval; SUV, standardized uptake value; GGO, ground-glass opacity; $T D R$, tumor shadow disappearance rate; $B A C$, bronchioloalveolar carcinoma.

vessel, and lymph node involvement compared with GGO, TDR, and BAC. Surprisingly, maximum SUV obtained preoperatively could be a more reliable indicator for predicting tumor malignancy than BAC proportion obtained

TABLE 6. Logistic regression analysis of clinicopathologic factors for positive predictive values of pathologic nodal status

\begin{tabular}{lll}
\hline Factors & Odds ratio $(95 \%$ CI) & P value \\
\hline Model $1\left(R^{2}=0.146\right)$ & & \\
Age & $0.960(0.880-1.048)$ & .3613 \\
Gender & $0.530(0.086-3.279)$ & .4948 \\
CEA & $0.999(0.774-1.290)$ & .9936 \\
Maximum SUV & $1.709(1.088-2.684)$ & .0199 \\
Model 2 $\left(R^{2}=0.136\right)$ & & \\
Age & $0.949(0.866-1.039)$ & .2577 \\
Gender & $0.811(0.164-4.021)$ & .7977 \\
CEA & $1.026(0.813-1.295)$ & .8270 \\
GGO ratio & $0.961(0.920-1.005)$ & .0800 \\
Model 3 $\left(R^{2}=0.282\right)$ & & \\
Age & $0.948(0.858-1.047)$ & .2925 \\
Gender & $0.858(0.143-5.153)$ & .8669 \\
CEA & $1.013(0.751-1.367)$ & .9334 \\
TDR & $0.937(0.890-0.987)$ & .0136 \\
Model 4 $\left(R^{2}=0.208\right)$ & & \\
Age & $0.960(0.869-1.061)$ & .4247 \\
Gender & $0.589(0.113-3.070)$ & .5295 \\
CEA & $0.989(0.776-1.261)$ & .9312 \\
Pathologic BAC ratio & $0.958(0.920-0.998)$ & .0375 \\
\hline
\end{tabular}

Category for gender and continuous variables for age, CEA, maximum SUV, GGO ratio, TDR, and BAC ratio. Cl, Confidence interval; $C E A$, carcinoembryonic antigen; SUV, standardized uptake value; GGO, ground-glass opacity; $T D R$, tumor shadow disappearance rate; $B A C$, bronchioloalveolar carcinoma. postoperatively. These results suggested that maximum SUV is a reasonable surrogate marker of adenocarcinoma invasiveness and that FDG-PET/CT could be a powerful prognostic tool with which to identify patients at high and low risk of recurrence after complete resection of small adenocarcinomas with or without a BAC component.

The relationships between the pathologic $\mathrm{BAC}$ ratio and radiographic maximum SUV, GGO ratio, and TDR in small adenocarcinomas are of interest in helping to understand the fundamental role of maximum SUV. The extent of both the GGO area and TDR closely correlated with that of BAC growth. However, we emphasize that maximum SUV, in comparison with the GGO ratio and TDR, was less associated with the BAC proportion. This discrepancy can be essential when considering the underlying significance of maximum SUV. That is, graphic maximum SUV, unlike the GGO ratio or TDR, is less able to reflect the pathologic $\mathrm{BAC}$ proportion and might be an imperative predictor for the grade of tumor malignancy independently of the BAC component.

The current relative decrease in the size of resected lung tumors allows a choice of various surgical options. ${ }^{2}$ Choosing radical sublobar resection for patients who could tolerate lobectomy requires better methods of preoperatively distinguishing early, indolent from advanced, aggressive cancers. However, no absolutely accurate preoperative indicators of tumor behavior have been defined, although several studies have reported that the GGO ratio and TDR on HR-CT as well as tumor size and histology are useful. $^{3-5,17}$ Our series showed that maximum SUV on PET/CT is a potentially promising parameter for determining an indication for radical sublobar resection. We also examined the relationship between maximum SUV and 


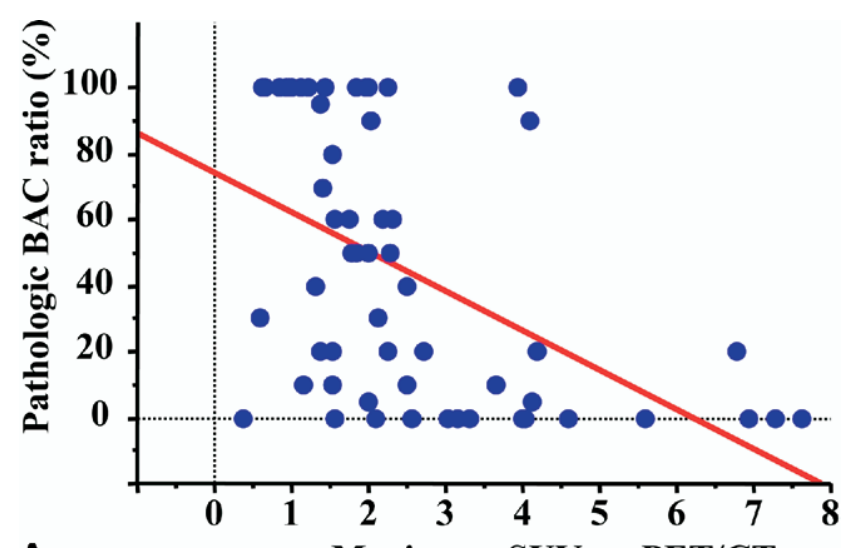

A
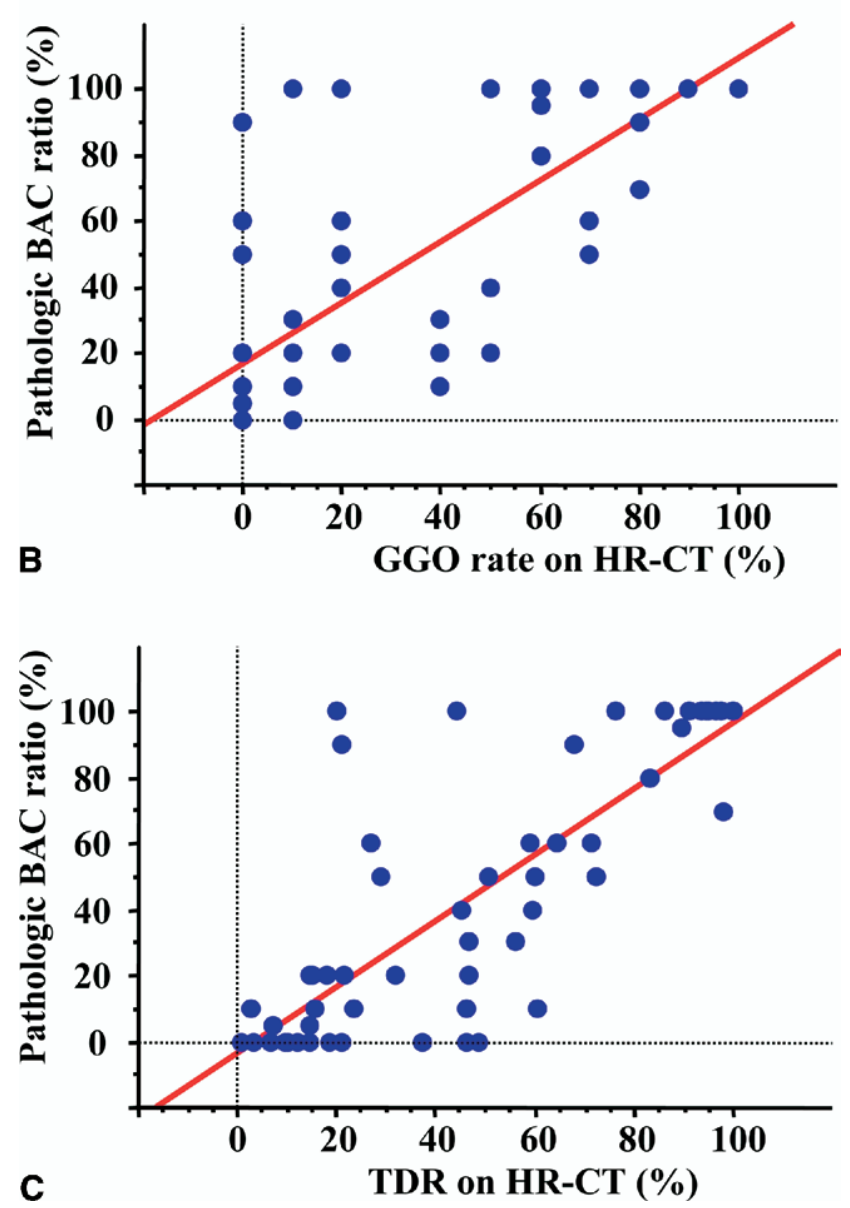

Figure 1. Regression parameters of radiographic findings and pathologic results in cT1NOMO adenocarcinomas. A, Correlation between maximum SUV on FDG-PET/CT and pathologic BAC ratio $\left(R^{2}=0.245, P<.0001\right)$. B, Correlation between GGO ratio on HR-CT and pathologic BAC ratio $\left(R^{2}=0.554, P<.0001\right)$. C, Correlation between TDR on HR-CT and pathologic BAC ratio $\left(R^{2}\right.$ $=0.671, P<.0001)$.

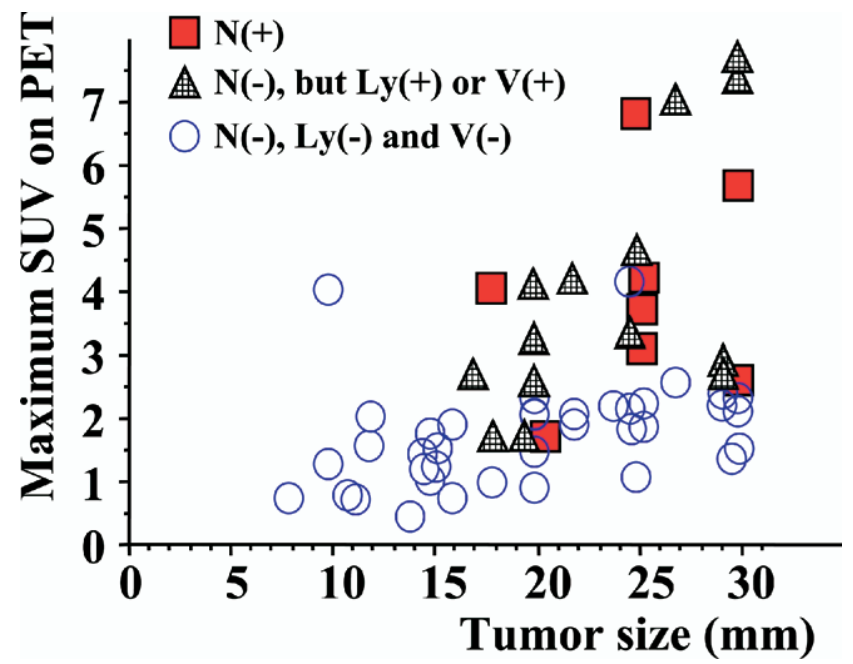

Figure 2. Relationship between tumor size and maximum SUV level in cT1NOMO adenocarcinomas. Squares, Nodal metastasis; triangles, no nodal metastasis but lymphatic or vascular invasion; circles, no findings of nodal metastasis, lymphatic and vascular invasion.

tumor size in cT1N0M0 adenocarcinomas (Figure 2). We found that the larger the tumor, the higher the maximum SUV, and both of these factors can predict tumor invasiveness and nodal metastases. Thus, FDG-PET/CT in addition to HR-CT, may be significantly useful for an indication of radical sublobar resection and a reduction in nodal dissection for surgically treating small adenocarcinomas, which requires further confirmation in a large cohort.

\section{References}

1. Ginsberg RJ, Rubinstein LV. Randomized trial of lobectomy versus limited resection for T1 N0 non-small cell lung cancer. Lung Cancer Study Group. Ann Thorac Surg. 1995;60:615-22.

2. Okada M, Koike T, Higashiyama M, Yamato Y, Kodama K, Tsubota N. Radical sublobar resection for small-sized non-small cell lung cancer: a multicenter study. J Thorac Cardiovasc Surg. 2006;132: 769-75.

3. Okada M, Nishio W, Sakamoto T, Uchino K, Yuki T, Nakagawa M, et al. Effect of tumor size on prognosis in patients with non-small cell lung cancer: the role of segmentectomy as a type of lesser resection. J Thorac Cardiovasc Surg. 2005;129:87-93.

4. Okada M, Nishio W, Sakamoto T, Uchino K, Hanioka K, Ohbayashi $\mathrm{C}$, et al. Correlation between computed tomographic findings, bronchioloalveolar carcinoma component, and biologic behavior of smallsized lung adenocarcinomas. J Thorac Cardiovasc Surg. 2004;127: 857-61.

5. Okada M, Nishio W, Sakamoto T, Uchino K, Tsubota N. Discrepancy of computed tomographic image between lung and mediastinal windows as a prognostic implication in small lung adenocarcinoma. Ann Thorac Surg. 2003;76:1828-32.

6. Nomori H, Watanage K, Ohtsuka T, Naruke T, Suemasu K, Kobayashi $\mathrm{T}$, et al. Fluorine 18-tagged fluorodeoxyglucose positron emission tomographic scanning to predict lymph node metastasis, invasiveness, or both, in clinical T1 N0 M0 lung adenocarcinoma. J Thorac Cardiovasc Surg. 2004;128:396-401. 
7. Cerfolio RJ, Ojha B, Bryant AS, Bass CS, Bartalucci AA, Mountz JM. The role of FGD-PET scan in staging patients with non small cell carcinoma. Ann Thorac Surg. 2003;76:861-6.

8. Cerfolio RJ, Bryant AS, Ojha B. Restaging patients with N2 (stage IIIa) non-small cell lung cancer after neoadjuvant chemoradiotherapy: a prospective study. J Thorac Cardiovasc Surg. 2006;131:1229-35.

9. McCain TW, Dunagan DP, Chin R Jr, Oaks T, Harkness BA, Haponik $\mathrm{EF}$. The usefulness of positron emission tomography in evaluating patients for pulmonary malignancies. Chest. 2000;118:1610-1615.

10. Pieterman RM, van Putten JW, Meuzelaar JJ, Mooyaart EL, Vaalburg W, Koeter GH, et al. Preoperative staging of non-small cell lung cancer with positron emission tomography. N Engl J Med. 2000;343: 254-61.

11. Cerfolio RJ, Bryant AS, Ohja B, Bartolucci AA. The maximum standardized uptake values on positron emission tomography of a non-small cell lung cancer predict stage, recurrence, and survival. J Thorac Cardiovasc Surg. 2005;130:151-9.

12. Dhital K, Saunders CAB, Seed PT, O'Doherty MJ, Dussek J. $\left[{ }^{18} \mathrm{~F}\right] \mathrm{Flu}-$ orodeoxyglucose positron emission tomography and its prognostic value in lung cancer. Eur J Cardiothorac Surg. 2000;18:425-8.

13. Vansteenkiste J, Fischer BM, Dooms C, Mortensen J. Positronemission tomography in prognostic and therapeutic assessment of lung cancer: systematic review. Lancet Oncol. 2004;5:531-40.

14. Ahuja V, Coleman RE, Herndon J, Patz EF Jr. The prognostic significance of fluorodeoxyglucose positron emission tomography imaging for patients with nonsmall cell lung carcinoma. Cancer. 1998;83: 918-24.

15. Mountain CF. Revisions in the International System for Staging Lung Cancer. Chest. 1997;111:1710-7.
16. Lee JR, Madsen MT, Bushnel D, Menda Y. A threshold method to improve standardized uptake value reproducibility. Nucl Med Commun. 2000;21:685-90.

17. Takamochi K, Nagai K, Yoshida J, Suzuki K, Ohde Y, Nishimura $\mathrm{M}$, et al. Pathologic N0 status in pulmonary adenocarcinoma is predictable by combining serum carcinoembryonic antigen level and computed tomographic findings. J Thorac Cardiovasc Surg. 2001;122:325-30

18. Noguchi M, Morikawa A, Kawasaki M, Matsuno Y, Yamada T, Hirohashi S, et al. Small adenocarcinoma of the lung. Histologic characteristics and prognosis. Cancer. 1995;75:2844-52.

19. Goldstein NS, Mani A, Chmielewski G, Welsh R, Pursel S. Prognostic factors in T1 N0 M0 adenocarcinomas and bronchioloalveolar carcinomas of the lung. Am J Clin Pathol. 1999;112:391-402.

20. Yap CS, Schiepers C, Fishbein MC, Phelps ME, Czernin J. FDG-PET imaging in lung cancer: how sensitive is it for bronchioloalveolar carcinoma? Eur J Nucl Med Mol Imaging. 2002;29:1166-73.

21. Heyneman LE, Patz EF. PET imaging in patients with bronchioloalveolar cell carcinoma. Lung Cancer. 2002;38:261-6.

22. Okada M, Nishio W, Sakamoto T, Uchino K, Yuki T, Nakagawa A, et al. Prognostic significance of perioperative serum carcinoembryonic antigen in non-small cell lung cancer: analysis of 1,000 consecutive resections for clinical stage I disease. Ann Thorac Surg. 2004;78: 216-21.

23. Okada M, Nishio W, Sakamoto T, Uchino K, Yuki T, Nakagawa A, et al. Effect of histologic type and smoking status on interpretation of serum carcinoembryonic antigen value in non-small cell lung carcinoma. Ann Thorac Surg. 2004;78:1004-9. 